\title{
Group Interventions for Children and Adolescents with Emotional Problems: A Review of The Literature
}

\author{
Preethi Anne Ninan ${ }^{1}$, Uma Hirisave ${ }^{2}$, Satish Chandra Girimaji ${ }^{3}$ \\ ${ }^{1} \mathrm{PhD}$ Scholar, Department of Clinical Psychology \\ ${ }^{2}$ Professor, Department of Clinical Psychology \\ ${ }^{3}$ Senior Professor, Department of Child and Adolescent Psychiatry \\ National Institute of Mental Health and Neurosciences (NIMHANS), Bangalore \\ Corresponding author: Preethi Anne Ninan \\ Email - anneninan@gmail.com
}

\begin{abstract}
Group interventions play an important role in therapeutic programs with children and adolescents with emotional problems or disorders, which have high rates of comorbidity and can persist into adulthood. The aim of this study was to review literature in the area of group interventions for children and adolescents, particularly with regard to emotional problems. Relevant studies were reviewed with regard to methodology, findings and implications. Gaps in existing literature, particularly within the Indian context, have been highlighted. Recent literature trends towards the use of brief closed group interventions, the use of transdiagnostic protocols, and varying levels of parental involvement. The implications of carrying out group interventions in various settings have been described.
\end{abstract}

Keywords: Group intervention, emotional problems, childhood, adolescence.

(Paper received $-12^{\text {th }}$ December 2018 , Peer review completed $-5^{\text {th }}$ January 2019)

(Accepted $-6^{\text {th }}$ January 2019)

\section{INTRODUCTION}

The periods of childhood and adolescence lay the early foundations for further development, well-being and satisfaction. Conversely, problems that arise in these periods could also contribute to further difficulties and maladjustment. Thus, there is significant scope for both promotive and therapeutic mental health interventions during these developmental stages.

The term emotional disorders [1] is synonymous with internalising disorders in the paediatric population, which is used to refer to anxiety, depression and somatic complaints which have high comorbidity rates [2]. A review of epidemiological work carried out in community surveys across the world [3] summarises that approximately one-fourth of youth experience a mental disorder over the previous year, while onethird experience one over their lifetime. Of these, anxiety disorders are the most frequent, and behaviour, mood and substance use disorders follow. Among the cases presenting to a Child and Adolescent Psychiatry clinic in North India, diagnoses of neurotic and stress related disorders constituted $16.4 \%$ $18.5 \%$ [4].

Meta-analytic research findings on psychotherapy with this age group [5-6] indicate effect sizes which are comparable to that for empirically-based adult treatments. Treatments which have emerged as wellestablished with children and adolescents [7] include cognitive-behavioural therapy, exposure, modelling, CBT with parents, education, and CBT with medication. A recent emphasis is also on transdiagnostic approaches to psychotherapy with this population [8], such as a 'transdiagnostic internalising toolbox for paediatric practice' [2], as well as principle-guided approaches [9]. 


\section{Application of group interventions for children and adolescents in clinical settings}

Group psychotherapy with adults had its origins in the early work of Pratt, Moreno and Yalom in the $20^{\text {th }}$ century. Pioneers in the field emphasised the role of therapeutic factors in groups, which include that of instilling hope, universality, imparting information, altruism, corrective recapitulation of the primary family group, developing socialising techniques, imitative behaviour, interpersonal learning, group cohesiveness, catharsis and existential factors [10].

Shechtman [11] distinguishes between three types of groups used with children and adolescents: guidance or educational groups (primary level prevention groups), counselling groups (secondary level prevention groups), and group psychotherapy (tertiary level prevention groups). Kymissis [12] describes the main indicators across groups with adolescents as those whose problems arise in the context of peer relationships, whose individual therapy may be stagnant or cannot begin individual therapy for various reasons (financial and otherwise), and who lack social skills. On the other hand, key contra-indicators include those who exhibit psychotic, violent, impulse-control, or substance use symptoms, are unmanageable or may tend to monopolise, become scapegoats or bullies in the group.

The benefits of groups can include efficiency in time, space, staff and finances; ongoing assessments as individuals can be observed in interactions with peers with regard to use of skills; the availability of social comparison and learning; and motivation to engage and be active [13]. Groups with adolescents may be characterised by more feedback and confrontation as compared to groups with children, while bringing challenges of resistance to authority, inflexibility and intolerance [11]. Research recommends the use of same-age groups to ensure similarity in developmental tasks, interests and abilities; and same-sex groups to exclude the possible tension that could arise between genders [11-12].

The recent decades have seen highly specialised group intervention modules or packages developed in the western settings, for specific problems such as anxiety disorders, depression, ADHD, anger and aggression, post-traumatic stress disorder (PTSD), substance abuse, deliberate self-harm, eating disorders and so on. Such programs may be applicable to groups which are homogenous in diagnoses, which may be possible only in randomised controlled trials (RCTs), but are 'the exception' in most clinical settings [14], where comorbidity amongst disorders is common. This is especially true with regard to anxiety and depressive disorders in children and adolescents, where comorbidity can occur through multiple developmental pathways [15]. Groups can be conducted in inpatient or outpatient settings; those in an outpatient clinical setting have the advantages of being able to recruit larger numbers of participants and offering a setting very similar to school [14].

\section{FINDINGS FROM STUDIES ON GROUP INTERVENTIONS FOR CHILDREN AND ADOLESCENTS}

\section{Method of literature search}

The purpose of this review was to identify and examine the literature on group interventions with children and adolescents, with a specific focus and outlook towards those with emotional difficulties. This was done through a literature search during the period of 2015-2018, with online searches on databases or search engines including PubMed, Proquest and Ebsco. Keywords used included 'group therapy / group psychotherapy / group intervention / group work with children', and similar terms with the word 'adolescents'. Additional searches were made for meta-analytic studies and those from the Indian context. Efforts were made to trace articles cited in books and chapters on the topic, and these were reviewed if relevant. Journal articles were explored through abstracts for relevance. Following this, full-length articles were reviewed if these highlighted or explored certain key aspects of conducting group interventions with this population, or if these used groups with children/adolescents with emotional problems or disorders.

The first section of the review will be on studies using group interventions with children and adolescents across target groups and formats, before moving to studies with a specific focus on emotional problems or disorders. A few studies examining processes in group interventions will be examined. Group interventions in the Indian context will then be analysed, and meta-analytic reviews in this area of study will be elucidated on. The paper ends with a summary of points gleaned from across the studies, as well as with implications for clinical practice and research. 


\section{Group intervention studies across formats and target groups}

The studies reviewed in this section were chosen as these highlight certain key or unique aspects with regard to the utility of groups for children and adolescents. These studies were conducted across various presenting problems and utilising diverse formats.

An early study by Abraham, Lepisto and Schultz [16] explored the perceptions of adolescents following their experience in structured group therapy and process group therapy. The study recruited 82 inpatient adolescents with psychotic, affective, behavioural problems or personality disorders. All were simultaneously undergoing speciality groups (12 weekly sessions based on common themes of divorce/adoption/sexual abuse, etc.), and process groups running over 10 months which were more 'interpersonally oriented'. Adolescents' self-report indicated that all preferred individual therapy over all other forms of treatment, including groups and family therapy. Process groups were preferred with regard to relating with peers and staff, but speciality groups were preferred with regard to understanding and dealing with the problem. This is one of the few studies identified which was able to compare experiences in various groups and also between group and individual therapy simultaneously; this format may not be possible in routine clinical settings, but rather in long-term residential settings.

Another study from an inpatient setting by Snyder, Kymissis and Kessler [17], looked at the efficacy of brief group therapy in reducing anger and aggression in adolescents. Adolescents with varied diagnoses were seen, if they scored atleast $75 \%$ or higher on the Trait Anger scale of the State-Trait Anger Expression Inventory (STAXI). While the treatment group received 4 sessions focusing on anger management, the control group were shown relevant psychoeducational videos. Post-treatment, the treatment groups' selfreported anger had decreased, while the control groups' had increased slightly. Merits of this study include the brevity of the intervention which proved efficacious, formation of groups based on a common problem even with heterogeneity in diagnosis, use of blind ratings by teachers and nurses, and the emphasis on generalisation and maintenance of skills.

Most research on the topic involves closed groups with the same group composition from beginning to end. This is possibly because of the inherent difficulties in conducting research with open groups, where members enter and leave the group at different time points. Only one study could be identified [18] which compared open and closed group interventions; this was done with adolescent girls who had encountered sexual abuse. While 13 girls experienced an open group intervention in comparison to 13 girls in a control condition (no treatment), 29 girls experienced a closed group intervention. Both open and closed group interventions involved 20 weekly sessions with 'psychoeducational content'. Not many details are included about the treatment manual, although it is evident that the intervention included structure while facilitating group processes to emerge around common experiences. Results indicated that both group intervention formats had similar significant gains. The exact nature of open groups is not clearly specified, which would have been relevant to understanding how to conduct these groups in clinical practice.

A program titled Exploring Together [19] addressed children and early adolescents with externalising behaviour problems in Australia. The treatment condition consisted of 106 children in groups of 4-8 participants for 8-10 weeks, each weekly session being 1.5 hours long, in comparison to a waitlist condition. Each session involved the children's group focusing on anger management, social and problemsolving skills (1 hour); the parents group conducted simultaneously aimed at parents-skills training (PST) and their difficulties; thereafter, both were seen together ( 0.5 hour) focusing on their interactions. Results indicated that children's behaviour problems reduced and social skills improved at home, and these gains were maintained at follow-up. The significance of this study lies in the use of concurrent parent and child groups as well as combined groups, and the brief nature of the program coinciding with the length of the school term.

A study distinctive in its format [20] was conducted with 63 adolescents, referred after an act of deliberate self-harm (DSH) and with at least one previous act of DSH in the last year. Participants were randomly allotted to either group therapy with routine care, or routine care alone; the groups were conducted according to a manualised program titled 'Developmental group psychotherapy'. This involved six acute-care group sessions, while the longer-term group therapy phase that followed was held weekly as long as perceived necessary by the adolescent. Adolescents in the intervention condition reported fewer DSH attempts and better school attendance than the comparison condition, although there were no significant 
effects on self-reported depression or suicidal thinking. Significant points to be gleaned from the study include its unique format and flexibility in number of sessions, and the nature of the intervention which appears eclectic in approach.

Other articles have similarly focused on different presenting problems or target groups; for instance, with children and adolescents with 'learning disabilities' [21]; Scholten et al. [22] through the use of a psychosocial group intervention, titled 'Op Koers' ('On Track') for children with chronic illnesses; Cone, Golden and Hall [23] through a short-term cognitive-behavioural group therapy (CBGT) for adolescents with attachment difficulties, residing in adoptive/foster/group homes; with juvenile offenders [24]; for students who engage in proactive aggressive behaviours or bullying [25]; for adolescents who have witnessed or experienced complex traumas [26]; online groups, such as for adolescents having received treatment for cancer [27]; through support groups for children who have parents with a psychiatric illness [28]; and so on. Various cognitive-behavioural group interventions for different presenting problems are presented in the Handbook of Cognitive-Behavioural Group Therapy with children and adolescents [29]. Research has also examined parent-child groups [30-32].

Although not exhaustive in its coverage, this section focused on a few studies which are notable for the design or intervention model that was utilised. The studies that follow, specifically involve group interventions targeting emotional disorders or difficulties.

\section{Group interventions with children and adolescents: a focus on emotional problems}

The studies available on group interventions with children and adolescents with emotional problems were found to involve the development and utilisation of treatment protocols, targeting either emotional disorders or emotional problems, and applied in a community setting or clinical setting. Some of these treatment protocols are seen in the studies reviewed below.

With regard to depression, one of the widely recognised protocols is Coping with Depression-Adolescents (CWD-A). Clarke, Rohde, Lewinsohn, Hops and Seeley [33] replicated the findings of efficacy of this program with 123 adolescents diagnosed with major depression or dysthymia. The acute phase included either adolescent group therapy of 16 hours, adolescent groups with a parent group, and the waitlist control group, while the maintenance phase randomised all treatment completers $(n=96)$ to booster session groups. Results indicated that the two treatment groups were superior to the waitlist group. The value of this study lies in the large sample, the emphasis on booster sessions and maintenance of gains, and the involvement of parents. The authors also suggest alternatives to booster sessions: for instance, telephone calls, or booster sessions only for adolescents with a higher risk of recurrence of depression. Other adaptations of the same program have also been found, including a school adaptation [34].

Straub et al. [35] carried out a pilot study to study the feasibility, efficacy, and safety (suicidal ideation) of a brief CBGT program for adolescents with depression in an outpatient setting. It was conducted with 15 adolescents in three groups of 4 to 6 participants, using the "Manualised Intervention to Cope with depressive symptoms, Help strengthen resources, and Improve emotion regulation" (MICHI) intervention of 5 sessions. Results indicated good feasibility, in terms of attendance, overall satisfaction, and adherence rate with the manual. Depressive symptoms, suicidal ideation and quality of life improved; however, there were no significant changes in parents' rating of adolescents' symptoms or functioning. The strength of this pilot study lies in the clearly-defined screening process, and the findings that suggest that a brief format of 5 sessions can bring effective change. A description of the full study and its allied objectives is found in Straub [36]. The effects of this program on neural network connectivities in adolescents have been reported [37], by comparing 19 adolescents with depression to 19 healthy controls. The treatment group received the 5-session program described above. Resting-state scans done prior to therapy, indicated that connectivity of the amygdala and subgenual ACC with regions of the default mode network, was stronger in patients as compared to healthy controls, and this decreased after intervention; whereas connectivity of the affective regions and those processing cognitions and salient stimuli, improved after intervention.

The use of interpersonal psychotherapy (IPT) for adolescent depression is well-recognised, and its application in a group format has been explored in a few studies. Mufson, Gallagher, Dorta and Young [38] comprehensively describe the session content of a group adaptation of interpersonal psychotherapy for depressed, non-bipolar disorder adolescents, and the rationale of the same in allowing adolescents to 
practice skills learnt in this environment which acts as a 'social laboratory', reducing their sense of isolation, and using collaborative efforts of the group to motivate adolescents and find other solutions. Their adaptation consists of two intensive pre-group individual sessions followed by 12 group sessions, with individual parent-adolescent sessions mid-way and at the end of treatment.

Further studies have explored the use of such group formats of IPT. For instance, O'Shea, Spence and Donovan [39] compared and evaluated the effects of individual and group IPT for depressed adolescents. Their study included 39 adolescents, with 20 adolescents in three groups in the group therapy condition, and 19 in the individual therapy condition, both receiving similar content through 12 sessions and 4 maintenance sessions. Adolescents in both formats showed significant improvements on measures of depression, anxiety, youth-reported internalising problems, and clinician-rated functioning. Only the measure of parent-reported internalising problems evidenced a significant difference between formats in the completers sample, with individual therapy showing greater improvements. Another study done in Puerto Rico [40] compared 12 sessions of both individual and group formats of CBT and IPT (as 'probably efficacious treatments' for depressed adolescents) for 112 adolescents. $62 \%$ in the CBT format, and $57 \%$ in IPT, moved from clinical to normative scores on CDI, indicative of clinically significant change. No difference was seen between individual and group formats, although individual formats had some increased efficacy in terms of effect size. The paper is a noteworthy example of how well-accepted manuals can be adapted according to the culture, as it has a clear description of the Latino values that the adaptation was linked to.

Another noteworthy study from outside the western context addressing depression in adolescents, was that of Bella-Awusah, Ani, Ajuwon, and Omigbodun [41] in Nigeria. This used a school-based CBT program of 5 sessions among 20 children with depression as a single group, compared to 20 waitlist controls. The treatment group showed significantly lower depressive symptoms at post intervention and 16 weeks post treatment. What is commendable about this study is the inclusion of various culture-specific components, including religious and cultural coping strategies, local metaphors and analogies. The study is also portrayed as an exemplar of a CBT-based intervention in a lower and middle-income country (LAMIC). However, the program involved a single group of 20 children from the same school, which may not have facilitated the development of group dynamics.

Programs have also been developed for anxiety disorders as a whole, or specific anxiety disorders. An RCT by Manassis et al. [42] used the Coping Cat program, comparing individual and group therapy with children (aged 8-12 years) with different Axis I anxiety disorders. The study randomly assigned 78 children to either individual or group therapy, both involving parents intensively. Both formats received 12 sessions of therapy from Kendall's Coping Cat Workbook, adapted for individual and group therapy (titled as the Coping Bear Workbook). Regardless of the condition, there was significantly decreased anxiety and improved global functioning at the end of the study. The study was thus able to compare individual and group interventions and demonstrated their relatively equivalent contributions. The authors however mention that children with higher social anxiety might do better in individual therapy, and that children with comorbid depression could also receive therapeutic components of mood regulation, which highlights the value of transdiagnostic approaches.

The Coping Cat program has also been used in other settings, including in the Chinese context by Lau, Chan, $\mathrm{Li}$ and $\mathrm{Au}$ [43], and in the Brazilian context by de Souza et al. [44]. The first involved 45 children between ages 6-11 years, diagnosed with an anxiety disorder or problems excluding specific phobias. The participants received nine two-hour weekly sessions in groups of 7 to 9 children. Changes, termed as 'culturally relevant', included involving parents as 'collaborators', taking parents/children's questions prior to ending sessions, telephoning after missed sessions to avoid dropouts, including Chinese concepts in therapy, and offering psycho-education. Results indicated that children in the treatment condition showed a significant improvement in terms of anxiety symptoms as compared to the waitlist condition. The two variables of anxiety cognitions and coping, mediated the association between treatment status and outcomes. The contributions of this study lie in providing further evidence for this program in a nonWestern scenario, and the culture-specific adaptations that could be made.

The study by de Souza et al. [44] also used the group version of the Coping Cat for children aged 10-13 years. The authors cite it as an example of a CBGT for children with anxiety disorders in the community 
in a lower and middle income country (LaMIC). The open clinical trial saw 20 completers of 28 children recruited, who participated in 14 group sessions and 2 concurrent parent sessions, with children showing an improvement on all measures including of anxiety, functioning, and externalising symptoms; however, depressive symptoms and quality of life did not significantly improve. While an important study in terms of the use of CBGT in the community and in a non-western setting, it lacked follow-up information, as well as any information on adaptations made for the context, and had a small number of completers for analysis.

Another study along similar lines targeting anxiety disorders [45] used an RCT implementing a cognitivebehavioural group therapy program termed as the FRIENDS program (Feeling worried? Relax and feel good; Inner thoughts; Explore plans; Nice work so reward yourself; Don't forget to practice; Stay calm, you know how to cope now). This involved 71 children aged 6-10 years in 10 treatment groups, for 10 weekly sessions with 2 booster sessions, besides an intensive parent component. At post-intervention, $69 \%$ of the children were diagnosis-free, as compared to $6 \%$ in the control group, besides high rates of treatment satisfaction from both parents and children. However, booster session attendance was very low, and the authors suggest these sessions may be recommended only for those who report of difficulties in skills generalisation or maintenance. The paper also states that a parallel version for adolescents aged 12-16 years has been developed.

One study identified which targeted a specific anxiety disorder, is that of Hayward et al. [46], who examined the efficacy of cognitive-behavioural group therapy for adolescent girls with social phobia through a pilot study. Their 16-week group intervention was implemented for 12 adolescents, as compared to 23 untreated controls with social phobia, and 18 adolescents without social phobia. The sessions focused on psycho-education, skills training, and exposure, besides other elements. The results indicated a significant improvement on the primary outcome measures for the treatment group. At post treatment, $55 \%$ in the treatment group, as compared to $96 \%$ in control, still had Social Phobia, but this difference was no longer present at the one-year follow-up. The authors also highlight the 'synergistic' relationship between social phobia and depression in this study, which lends further credibility to studies which utilise a transdiagnostic approach towards anxiety and depressive disorders.

Amongst the transdiagnostic cognitive-behavioural approaches is that of the Unified Protocol for the treatment of emotional disorders in children and adolescents. Bilek and Ehrenreich-May [47] utilised the group protocol of the same, titled the Emotion Detectives treatment protocol, in working with 22 children aged 7-12 years with primary anxiety disorders and comorbid depressive symptoms. In line with the Unified Protocol, it used core principles of altering antecedent cognitive appraisals, preventing emotional avoidance, and modifying behavioural action tendencies. The treatment protocol of 15 sessions (further described in Ehrenreich-May, Bilek, Queen \& Rodriguez [48]), included content on awareness of emotions and acceptance of emotional states, cognitive appraisal, problem-solving skills, behaviour activation, exposure and parent training. Results indicated an improvement in child-reported anxiety symptoms, parent-reported depressive symptoms, and clinician-rated symptom severity. However, children with a primary depressive disorder diagnosis were not included, and being a feasibility study, involved a small sample and did not include a control group. The adolescent version of the Unified Protocol has been examined in various studies, such as in Ehrenreich-May, Rosenfield, Queen, Kennedy, Remmes and Barlow [49], which however is in an individual therapy format.

Another transdiagnostic protocol is that of the Cool Kids Adolescent Anxiety and Depression program, developed at the Centre for Emotional Health Clinic, Macquarie University, Australia. Varied forms of the Cool Kids program exist, and Rapee [50] elaborates on what appears to be an early version of the program for anxiety disorders, tested as a nine session program in groups, involving 95 children (aged 7 to 16 years), with major components of cognitive restructuring, exposure to fear-related stimuli and teaching the parents management techniques. The treated children improved compared to waitlist children, on measures of anxiety. Perini, Wuthrich and Rapee [51], in their commentary on the use of the Cool Kids in Denmark, also succinctly summarise the various versions including for anxiety alone, for anxiety and depression, school-based and outreach programs, the various formats of the program such as individual and group formats, and the involvement of parents to varying degrees. The Cool Kids Adolescent Anxiety and Depression program manual [52] includes components in 16 sessions, with a focus on psychoeducation; 
thought-feeling link, realistic thinking and working on core beliefs; exposure; managing emotions; behavioural experiments; problem-solving and its application to relationships; and a focus on assertiveness, teasing and stress management.

Other than cognitive-behavioural formats, alternate treatment protocols have also been used. For instance, narrative group therapy was used for boys, aged 10-11 years, with social phobia in Iran [53], randomly distributed to intervention versus waitlist groups. The male therapists were able to work only with boys, given the nation's policies regarding the same. The children received 14 narrative group sessions in the school setting, which were largely activity based. Detailed descriptions of the activities appear to target problem-solving through narrative means, besides a focus on emotions. The study reports a significant difference between treatment and waitlist group post-treatment and after one month; however, these reports are largely based on parental and teacher report, and the child's self-report would have added greater validity to results. The age range of participants also appears very limited, and there is little focus on group processes.

Another study, among the recent ones on group interventions with children and adolescents, is that of Salum et al. [54], comparing group CBT and attention bias modification (ABM) in children, aged 7-11 years, with anxiety disorders. The study was carried out in Brazil, and used a factorial design to compare group differences in children receiving G-CBT and ABM to their respective control interventions of Control Therapy and attention control training (ACT). Here, the group intervention received 10 weekly sessions based on the Friends for Life program, to increase social and emotional skills, resilience and reduce symptoms. However, the results from the study indicated significant improvements in all groups, with neither G-CBT nor ABM showing additional effects beyond that of control groups; in addition, the analyses pointed to higher dropout rates when G-CBT was combined with ABM.

\section{Process-related research on group interventions}

Most research on process factors in group interventions with this population, focuses on therapeutic factors. For instance, Brouzos, Vassilopoulos and Baourda [55] examined the endorsement of therapeutic factors in groups run for children with social anxiety in Greece. This was done though an analysis of answers on the Critical Incidents Questionnaire, reported by group members after each session, and categorised according to the stage of group development. Therapeutic factors most reported were learning from interpersonal actions and instillation of hope at the beginning; and guidance at the working and ending stages of the group, which the authors relate to the culture. Universality was unusually not reported at all, and acceptance reported only in the beginning. Vicarious learning was more prominent towards later stages, interpreted as important once cohesion increases and participants observe each other. Although the size of groups (mentioned as 9-16 in a group) and the fact that all group members were drawn from one school might have affected group processes, this study is an interesting example of the analysis of processes in groups and the manner in which such an exploration might be done.

Another qualitative insight into the experiences of adolescents in group therapy was undertaken through a dissertation [56], exploring the experiences of 8 such adolescents through semi-structured interviews. The adolescents had undergone three months of 'interpersonal group psychotherapy'. In their report of their experiences, they mentioned that they found the group as a place of gathering of hope, found support, received help and so on. While referring to the 'process' (or stages of the group), they referred to a 'nervous' phase, before settling in by session 3-4, later with deepening connections and sharing in the middle phase, and mixed emotions as they neared the end. Among their recommendations for future groups, they mentioned the importance of a comfortable physical space, small groups composed of 4-6 participants of similar age, and involving adolescents only if they are motivated to be there. Bussell [57] also explored group processes through the use of the Session Evaluation Form (SEF) for adolescents. The study found that the SEF, tapping into Group, Facilitator, Skills and Exploration Impact, could differentiate between groups in terms of impact of the therapy process. The use of the measure was taken further in the study by Garcia [58], including an investigation of its psychometric properties. 


\section{Studies on group interventions: the Indian scenario}

Studies in India with children and adolescents have been carried out primarily in non-clinical settings. An intervention study by Akoijam [59] tested the efficacy of counselling for adolescents with adjustment problems in the school setting, wherein the minimal contact group received a single group session with information on problem-solving skills. Among other significant results was that the minimal contact group also showed signs of improvement with the inputs from the group session. Anand [60] explored the contribution of Mindfulness-Based Stress Reduction (MBSR) conducted in groups, in reducing stress in adolescents, with results indicating significant differences in stress, well-being and adjustment in the intervention group as compared to the control group. However, a limited age group (aged 13-15 years) was taken, limiting the generalizability of results, and the program itself was promotive in nature.

Singhal [61-62] explored the efficacy of a school-based group coping skills program for adolescents with subclinical depression; the intervention condition of 10 groups, received 8 weekly sessions, while the control condition received one interactive session focusing on psychoeducation. The intervention group showed a clinically significant reduction in depressive symptoms, negative cognitions and academic stress, with increased social problem-solving and coping skills, while no corresponding changes were seen in the control group. This study was one of the few group intervention programs for adolescents in the Indian setting, which utilized the cognitive-behavioural format, and it incorporated common elements from wellrecognised manualized group interventions. However, it was carried out in a school setting, where adolescents are likely to have already known and been familiar with each other.

Banerjee [63] utilised the narrative approach with groups of adolescents in schools, using an adaptation of the manual of Monk and Winsdale. Adolescents aged 13-18 years, were seen in 6 groups over 6 sessions, with average group size being 31 adolescents. Participants' self-report revealed that the narrative approach aided in making them feel more confident in reaching goals, and in recognising their own ability to solve their own and others' problems. However, the large size of groups may have also limited group interactions and dynamics. Another study in the school setting [64, 65] developed and explored the efficacy of a group intervention for adolescent girls, aged 13-15 years. The 8-session group intervention with a class of female students, aimed at mental health promotion and development of self-awareness. Students showed improvements at post-intervention, although not all improvements were maintained at follow-up. All these studies reviewed so far, worked with adolescents considered 'at-risk' or with subclinical problems in a school setting, and results may not be generalizable to groups for those with psychiatric disorders in clinical settings.

Another recent study in this area in the Indian setting, by Easvaradoss and Cabral [66], used a CBGT program for adolescents from dual-earner families, with the rationale that such adolescents face distinctive difficulties. The sample consisted of 50 adolescents in 4 groups, each with 12-13 students, with 6 modules emphasising group dynamics, social skills, problem-solving skills, cognitive self-change and attribution retraining. Adolescents showed an increase in scores of emotional maturity, frustration tolerance, study involvement and parent and peer attachment after the intervention. This study is a fairly recent use of cognitive-behavioural group therapy in the Indian setting. However, a clearer description of the content of intervention is required.

Only one study utilising groups in a clinical setting could be noted in the Indian context [67] which examined the efficacy of transdiagnostic cognitive-behavioural group therapy, for an adolescent population with comorbid anxiety disorders and headache. The study utilised a 12-session group protocol, primarily developed after FGDs and examining related literature. It was carried out with 63 adolescents randomised to intervention versus treatment as usual (TAU). It is significant to note that groups were stratified by age (10-14, 15-19 years), although details about gender distribution are not specifically mentioned, leaving one to assume these groups were formed with both males and females. Results indicated that the treatment group showed significant improvement on state and trait anxiety scores, while TAU did not; although both conditions improved on measures of headache intensity and clinician-rated functioning, the treatment condition showed significantly greater improvement.

It is relevant to note the stages through which the transdiagnostic protocol was developed, with the inclusion of content on assertiveness training, emotion regulation and management of stress, based on themes from focused-group discussions with adolescents with such problems. However, details are not 
available regarding how content was made suitable for adolescents, in terms of means of delivery; whether the headache or anxiety disorder was the primary diagnosis; nor about group processes or facilitation of the same (besides that initial sessions sought to facilitate group cohesion and socialisation); and whether and how session content or process differed between groups stratified according to age. The session content described, appeared to primarily target anxiety symptoms, with less focus on the diagnosis of headache (other than monitoring of the headaches). In addition, adolescents with depression were deliberately excluded, but details about these adolescents are not given; this would have been relevant given high rates of comorbidity between anxiety and depression. In addition, a symptom-based measure of anxiety, rather than the State Trait Anxiety Inventory, would have been more suitable as an outcome measure. Nonetheless, this study points to the use of a transdiagnostic protocol for an emotional disorder in the Indian setting, and is highly significant in this regard.

\section{Meta analytic studies and reviews}

An important meta-analytic review of 56 articles by Hoag and Burlingame [68] specifically on child and adolescent (age 4 -18) group treatments, found that various forms of groups are more effective than waitlist or placebo control groups (with an effect size of .61), indicating that these children and adolescents were better off than $73 \%$ of those in control groups. The review indicated that studies of group interventions in school settings $(E S=.53)$ were significantly less effective than in clinical settings $(E S=$ 1.13); studies wherein experimenter allegiance was clear $(E S=.72)$ were more effective than therapies where allegiance was absent; groups oriented to counselling or therapy $(E S=.65)$ were more effective than psychoeducational groups $(\mathrm{ES}=.40)$; and that were relatively fewer studies with mood and anxiety disorders.

A recent meta-analytic review [69] compared and ranked the different types of psychotherapies aimed at acute anxiety disorders in children and adolescents. This network meta-analysis examined 101 randomised controlled trials with 6625 participants. The most significant finding from the analysis was in support of group CBT, in that only group cognitive behaviour therapy was significantly more effective at posttreatment than other psychotherapies (SMD range, -0.82 to -0.43) and all neutral control conditions (SMD range, -1.43 to -0.76 ). The study concludes that group CBT may be considered as the first choice of psychotherapy for anxiety disorders in children and adolescents, though group BT might be preferable in children.

\section{CONCLUSIONS}

This review paper sought to examine and analyse existing literature related to group interventions with children and adolescents, with a specific focus on those with emotional problems or emotional disorders. Literature has emphasised the developmental needs of this population, and the need for psychological interventions to be cognizant of their developmental abilities and mental health concerns that can arise during this period.

As noted from literature, group interventions confer many possible advantages and clinical benefits for children and adolescents and their families, as well as for professionals working with them. Not least amongst these are the advantages in terms of cost, time, and manpower efficiency, besides the benefits accrued to children and adolescents in being able to work alongside peers with similar difficulties.

With regard to theoretical frameworks, most recent studies reviewed from the Western setting utilised cognitive-behavioural group therapies (CBGTs), often with specific manualised programs, for children and adolescents with emotional problems. Other frameworks were also noted, including that of narrative therapies. These were largely developed for the clinical setting, at times used in community or school settings for those with sub-clinical problems or as a preventive approach. Adaptations of well-recognised manualised programs have been carried out in non-Western settings; some have outlined the process of adaptation and the inclusion of changes based on cultural values, with a focus on aspects such as feasibility and efficacy. The recent trend appears to be in favour of transdiagnostic or principle-guided approaches, which recognise the role of comorbidity in diagnoses. 
The majority of studies utilised closed groups, and it would therefore be important to understand the role of open groups for children and adolescents in clinical settings. Group interventions have been carried out with varying group sizes, ranging from 5 in a group to more than 30 , although most studies appear to endorse a maximum of 8 in a group. Studies often utilised homogenous samples in terms of diagnosis or presenting complaints, while at other times approaching children or adolescents through a common presenting problem such as anger, anxiety, or deliberate self-harm attempts, regardless of diagnosis. Particularly with regard to emotional problems or disorders, studies were carried out in outpatient clinical settings, which give individuals the opportunity to use these skills and inputs outside the therapy setting. The use of groups in outpatient settings may however restrict the chances of conducting multiple therapeutic interventions simultaneously, which may be possible in inpatient or residential settings. Many studies have moved towards the use of brief interventions, at times using 5 sessions, and have noted effectiveness of the same. Other studies have utilised booster sessions to facilitate maintenance of gains. A few have explored distinctive and flexible formats, including with varied levels of parental involvement. Some studies have referred to different versions of the group intervention based on age groups, for example with those in childhood, versus early, middle or late adolescence.

The results of such group interventions have primarily been explored through measures of symptom rating, reported by self, parents, or through clinician-rated measures. However, it is important to note that experiences of the group may not be accessible only through such measures. While most studies propose that children and adolescents can benefit from therapeutic processes of the group, they do not explore these group processes extensively. Thus, group experiences may need to be analysed through other means such as process ratings, adolescent and parent interviews, and therapist observations of session processes.

In the Indian setting, interventions using the group format are few and have largely been restricted to community or school settings. These studies in non-clinical settings can ensure homogeneity, for instance in terms of language or socio-economic status. Within these settings, group interventions have proven to be beneficial. Most such studies suggest or imply that similar programs need to be implemented in clinical settings. Only one study was found which utilised the same with adolescents in an outpatient clinical setting, through a transdiagnostic approach.

\section{Implications}

Given the advantages of group intervention approaches, the use of these programs can present a novel and clinically relevant approach to working with children and adolescents, specifically with emotional problems, in the Indian clinical setting. Such programs could be implemented with children or adolescents with a specific disorder, or with a common presenting problem regardless of diagnosis, or with transdiagnostic protocols aimed at highly comorbid conditions. In hospitals or private practice clinical settings, running group interventions during periods when academic demands may be less, such as during vacation periods or weekends, might ensure greater attendance and participation. In such contexts, it may be necessary for individual therapy and group interventions to complement each other, rather than either taking the place of the other. In a busy hospital setting, single-session groups can be a viable option to bring children or adolescents together on the day of their consultation, serving the purposes of providing psychoeducation and information on treatment options, allowing them to interact with other individuals with similar difficulties, as well as acting as a stepping-stone for more intensive group interventions. In general hospital or paediatric settings, children and adolescents presenting with primary medical issues along with other emotional difficulties, can be seen together in a group format to address mutual concerns. The involvement of parents in sessions is also extremely important, and the extent of involvement must be explored through clinical practice and research, ranging from a single session to sessions held concurrently with those of their children. Most importantly, the content of sessions, whether drawn from existing manualised programs or developed for the population at hand, must be adapted according to the specific age group in terms of activities and means of delivery, for instance through games, relevant examples, group discussions, role-plays, videos, and the like. 


\section{REFERENCES}

1. Moses EB, Barlow DH. A new unified treatment approach for emotional disorders based on emotion science. Curr Dir Psychol Sci 2006; 15(3):146-50.

2. Weersing VR, Rozenman MS, Maher-Bridge M, Campo JV. Anxiety, depression, and somatic distress: Developing a transdiagnostic internalizing toolbox for pediatric practice. Cogn Behav Pract 2012; 19(1):6882.

3. Merikangas KR, Nakamura EF, Kessler RC. Epidemiology of mental disorders in children and adolescents. Dialogues Clin Neurosci 2009;11(1):7-20.

4. Malhotra S, Biswas P, Sharan P, Grover S. Characteristics of patients visiting the Child \& Adolescent Psychiatric Clinic: A 26-year study from North India. J. Indian Assoc. Child Adolesc Ment Health 2007; 3(3):53-60.

5. Weisz JR, Weiss B, Han SS, Granger DA, Morton T. Effects of psychotherapy with children and adolescents revisited: a meta-analysis of treatment outcome studies. Psychol Bull 1995;117(3):450-68.

6. Weisz JR, Huey SJ, Weersing VR. Psychotherapy outcome research with children and adolescents. In: Ollendick TH, Prinz, RJ, editors. Advances in Clinical Child Psychology, Volume 20. Boston: Springer US; 1998. pp. 49-91.

7. Higa-McMillan CK, Francis SE, Rith-Najarian L, Chorpita BF. Evidence base update: 50 years of research on treatment for child and adolescent anxiety. J Clin Child Adolesc Psychol 2016;45(2):91-111.

8. Chu BC, editor. Translating transdiagnostic approaches to children and adolescents [Special series]. Cogn Behav Pract 2012;19:1-4.

9. Weisz J, Bearman SK, Santucci LC, Jensen-Doss A. Initial test of a principle-guided approach to transdiagnostic psychotherapy with children and adolescents. J Clin Child Adolesc Psychol 2017;46(1):4458.

10. Yalom ID, Leszcz M. The Theory and Practice of Group Psychotherapy. 5th ed. New York: Basic Books; 2005.

11. Shechtman Z. Group counselling and psychotherapy with children and adolescents: Current practice and research. In: DeLucia-Waack JL, Gerrity DA, Kalodner CR, Riva MT, editors. Handbook of Group Counselling and Psychotherapy. USA: Sage Publications; 2004.

12. Kymissis P. Group Psychotherapy with Adolescents. In: Kaplan HI, Sadock BJ, editors. Comprehensive group psychotherapy. Baltimore: Williams \& Wilkins Co.; 1993.

13. Stewart JL, Christner RW, Freeman A. An introduction to Cognitive-Behavior Group Therapy with youth. In: Christner RW, Stewart JL, Freeman A, editors. Handbook of Cognitive-Behavior Group Therapy with children and adolescents: Specific settings and presenting problems. New York: Routledge; 2007.

14. Friedberg RD. Group cognitive-behaviour therapy in outpatient settings. In: Christner RW, Stewart JL, Freeman A, editors. Handbook of cognitive-behavior group therapy with children and adolescents: Specific settings and presenting problems. New York: Routledge; 2007.

15. Cummings CM, Caporino NE, Kendall PC. Comorbidity of anxiety and depression in children and adolescents: 20 years after. Psychol Bull 2014;140(3):816-45.

16. Abraham PP, Lepisto BL, Schultz L. Adolescents' perceptions of process and specialty group therapy. Psychotherapy 1995;32(1):70-6.

17. Snyder KV, Kymissis P, Kessler K. Anger management for adolescents: Efficacy of brief group therapy. J Am Acad Child Adolesc Psychiatry1999;38(11):1409-16.

18. Tourigny M, Hébert M. Comparison of open versus closed group interventions for sexually abused adolescent girls. Violence Vict 2007;22(3):334-49.

19. Hemphill SA, Littlefield L. Evaluation of a short-term group therapy program for children with behavior problems and their parents. Behav Res Ther 2001;39(7):823-41.

20. Wood A, Trainor G, Rothwell J, Moore A, Harrington R. Randomized trial of group therapy for repeated deliberate self-harm in adolescents. J Am Acad Child Adolesc Psychiatry 2001;40(11):1246-53.

21. Mishna F, Muskat B. "I'm not the only one!" Group therapy with older children and adolescents who have learning disabilities. Int J Group Psychother 2004;54(4):455-76.

22. Scholten L, Willemen AM, Last BF, Maurice-Stam H, van Dijk EM, Ensink E, et al. Efficacy of psychosocial group intervention for children with chronic illness and their parents. Pediatrics 2013;131(4); 1196-203.

23. Cone JC, Golden JA, Hall CW. The effect of short-term cognitive-behavioral group therapy on adolescents with attachment difficulties. Behav Dev Bull 2009;15(1):29-35.

24. Choate LH, Manton J. Teen court counseling groups: Facilitating positive change for adolescents who are first-time juvenile offenders. J Special Group Work 2014;39(4):345-65. 
25. Fung AL, Gerstein LH, Yuichung C, Hutchison A. Cognitive-behavioral group therapy for Hong Kong students that engage in bullying. Revista de Cercetare si Interventie Sociala 2013;42:68-84.

26. Habib M, Labruna V, Newman J. Complex histories and complex presentations: Implementation of a manually-guided group treatment for traumatized adolescents. J Fam Viol 2013;28:717-28.

27. Sansom-Daly UM, Wakefield CE, Bryant RA, Butow P, Sawyer S, Patterson P, et al. Online group-based cognitive-behavioural therapy for adolescents and young adults after cancer treatment: a multicenter randomised controlled trial of Recapture Life-AYA. BMC Cancer 2012;12:339.

28. van Santvoort F, Hosman CMH, van Doesum KTM, Janssens JM. Effectiveness of preventive support groups for children of mentally ill or addicted parents: a randomized controlled trial. Eur Child Adolesc Psychiatry 2014;23:473-84.

29. Christner RW, Stewart J, Freeman A., editors. Handbook of cognitive-behavior group therapy with children and adolescents: Specific settings and presenting problems. New York: Routledge; 2007.

30. Barmish AJ, Kendall PC. Should parents be co-clients in cognitive-behavioral therapy for anxious youth?. J Clin Child Adolesc Psychol 2005;34(3):569-81.

31. Mendlowitz SL, Manassis K, Bradley S, Scapillato D, Miezitis S, Shaw BF. Cognitive- behavioral group treatments in childhood anxiety disorders: The role of parental involvement. J Am Acad Child Adolesc Psychiatry 1999;38(10):1223-9.

32. Ben-Amitay G, Rosental B, Toren P. Brief parent-child group therapy for childhood anxiety disorders: a developmental perspective on cognitive-behavioral group treatment. Int J Group Psychother 2010;60(3):389406.

33. Clarke GN, Rohde P, Lewinsohn PM, Hops H, Seeley JR. Cognitive-behavioral treatment of adolescent depression: efficacy of acute group treatment and booster sessions. J Am Acad Child Adolesc Psychiatry 1999;38(3):272-9.

34. Ruffolo MC, Fischer D. Using an evidence- based CBT group intervention model for adolescents with depressive symptoms: lessons learned from a school- based adaptation. Child Fam Soc Work 2009;14(2):189-97.

35. Straub J, Sproeber N, Plener PL, Fegert JM, Bonenberger M, Koelch MG. A brief cognitive-behavioural group therapy programme for the treatment of depression in adolescent outpatients: a pilot study. Child Adolesc Psychiatry Ment Health 2014;8(1):9-14.

36. Straub JF. Depression in adolescents: feasibility, efficacy, and neural correlates of a brief group psychotherapeutic treatment, along with insights on epidemiology, symptoms, and diagnostics (Doctoral dissertation). Ulm University, Germany: 2015.

37. Straub J, Metzger CD, Plener PL, Koelch MG, Groen G, Abler B. Successful group psychotherapy of depression in adolescents alters fronto-limbic resting-state connectivity. J Affect Disord 2017;209:135-9.

38. Mufson L, Gallagher T, Dorta KP, Young JF. A group adaptation of interpersonal psychotherapy for depressed adolescents. Am J Psychother 2004;58(2):220-37.

39. O'Shea G, Spence SH, Donovan CL. Group versus individual interpersonal psychotherapy for depressed adolescents. Behav Cogn Psychother 2015;43(1):1-19.

40. Rosselló J, Bernal G, Rivera-Medina C. Individual and group CBT and IPT for Puerto Rican adolescents with depressive symptoms. Cultur Divers Ethnic Minor Psychol 2008;14(3):234-45.

41. Bella- Awusah T, Ani C, Ajuwon A, Omigbodun O. Effectiveness of brief school- based, group cognitive behavioural therapy for depressed adolescents in south west Nigeria. Child Adolesc Ment Health 2016; 21(1):44-50.

42. Manassis K, Mendlowitz SL, Scapillato D, Avery D, Fiksenbaum L, Freire M, et al. Group and individual cognitive-behavioral therapy for childhood anxiety disorders: A randomized trial. J Am Acad Child Adolesc Psychiatry 2002;41(12):1423-30.

43. Lau WY, Chan CK, Li JC, Au TK. Effectiveness of group cognitive-behavioral treatment for childhood anxiety in community clinics. Behav Res Ther 2010;48(11):1067-77.

44. de Souza MA, Salum GA, Jarros RB, Isolan L, Davis R, Knijnik D, et al. Cognitive-behavioral group therapy for youths with anxiety disorders in the community: effectiveness in low and middle income countries. Behav Cogn Psychother 2013;41(3):2552-64.

45. Shortt AL, Barrett PM, Fox TL. Evaluating the FRIENDS program: A cognitive-behavioral group treatment for anxious children and their parents. J Clin Child Psychol 2001;30(4):525-35.

46. Hayward C, Varady S, Albano AM, Thienemann M, Henderson L, Schatzberg AF. Cognitive-behavioral group therapy for social phobia in female adolescents: Results of a pilot study. J Am Acad Child Adolesc Psychiatry 2000;39(6):721-6.

47. Bilek EL, Ehrenreich-May J. An open trial investigation of a transdiagnostic group treatment for children with anxiety and depressive symptoms. Behav Ther 2012;43(4):887-97. 
48. Ehrenreich-May J, Bilek EL, Queen AH, Hernandez Rodriguez J. A unified protocol for the group treatment of childhood anxiety and depression. Revista de Psicopatología y Psicología Clínica 2012;17(3):219-36.

49. Ehrenreich-May J, Rosenfield D, Queen AH, Kennedy SM, Remmes CS, Barlow DH. An initial waitlistcontrolled trial of the unified protocol for the treatment of emotional disorders in adolescents. J Anxiety Disord 2017;46:46-55.

50. Rapee RM. Group treatment of children with anxiety disorders: Outcome and predictors of treatment response. Aust J Psychol 2000;52(3):125-9.

51. Perini SJ, Wuthrich VM, Rapee RM. "Cool Kids" in Denmark: Commentary on a Cognitive-Behavioral Therapy Group for Anxious Youth. Pragmat Case Stud Psychother 2013;9(3):359-70.

52. Schniering CA, Rapee RM, Lyneham HJ, Wuthrich V, Hudson JL, Wignall A. The Cool Kids Adolescent Anxiety \& Depression Program Therapist Manual. Centre for Emotional Health, Macquarie University University: Sydney, Australia: 2006.

53. Looyeh MY, Kamali K, Ghasemi A, Tonawanik P. Treating social phobia in children through group narrative therapy. Art Psychother 2014;41(1):16-20.

54. Salum GA, Petersen CS, Jarros RB, Toazza R, DeSousa D, Borba LN, et al. Group Cognitive Behavioral Therapy and Attention Bias Modification for Childhood Anxiety Disorders: A Factorial Randomized Trial of Efficacy. J Child Adolesc Psychopharmacol 2018;28(9):620-30.

55. Brouzos A, Vassilopoulos SP, Baourda VC. Therapeutic factors and members' perception of co-leaders' attitudes in a psychoeducational group for Greek children with social anxiety. J Special Group Work 2015; 40(2):204-24.

56. Pingitore FR. Exploring the experiences of adolescents participating in interpersonal group psychotherapy (Doctoral dissertation). University of Rhode Island, USA; 2015.

57. Bussell JR. Exploring the role of therapy process and outcome in interventions that target adolescent identity and intimacy (Doctoral dissertation). Florida International University; 2000.

58. Garcia AJ. Investigating group processes in a positive youth development program for multi-problem youth (Doctoral dissertation). Florida International University; 2007.

59. Akoijam L. Efficacy of counselling for adolescent adjustment problems (Doctoral dissertation). National Institute of Mental Health and Neurosciences, Bangalore; 2003.

60. Anand U. Effectiveness of a mindfulness-based stress reduction program in reducing stress in adolescents (Doctoral dissertation). National Institute of Mental Health and Neurosciences, Bangalore; 2013.

61. Singhal M. Efficacy of a school-based group coping skills program for adolescents (Doctoral dissertation). National Institute of Mental Health and Neurosciences, Bangalore; 2014.

62. Singhal M, Munivenkatappa M, Kommu JV, Philip M. Efficacy of an indicated intervention program for Indian adolescents with subclinical depression. Asian J Psychiatry 2018;33:99-104.

63. Banerjee M. Narrative counselling for adolescents in school setting: An exploratory study (Dissertation). National Institute of Mental Health and Neurosciences, Bangalore; 2015.

64. John JM. Efficacy of group intervention module for adolescent girls on self-awareness (Doctoral dissertation). National Institute of Mental Health and Neurosciences, Bangalore; 2017.

65. John JM, Navneetham J, Nagendra HR. Development of a trans-disciplinary intervention module for adolescent girls on self-awareness. J Clin Diagn Res 2017;11(8):7-10.

66. Easvaradoss V, Cabral V. Impact of cognitive behavior group therapy on the psychological functioning of adolescents from dual earner families. Indian J Health Wellbeing 2015;6(2):177-80.

67. Sharma P, Mehta M, Sagar R. Efficacy of transdiagnostic cognitive-behavioral group therapy for anxiety disorders and headache in adolescents. J Anxiety Disord 2017;46:78-84.

68. Hoag MJ, Burlingame GM. Evaluating the effectiveness of child and adolescent group treatment: A metaanalytic review. J Clin Child Psychol 1997;26(3):234-46.

69. Zhou X, Zhang Y, Furukawa TA, Cuijpers P, Pu J, Weisz JR, et al. Different types and acceptability of psychotherapies for acute anxiety disorders in children and adolescents: a network meta-analysis. JAMA Psychiatry 2019;76(1):41-50.

Acknowledgements - Nil

Source of Funding - Nil

Conflict of Interest - Nil 